\title{
Abbauprodukte von Amiloridhydrochlorid
}

\author{
Klaus Görlitzer * ${ }^{1}$, Silke Huth ${ }^{1}$, \\ Edith Gößnitzer ${ }^{2}$ und Winfried Wendelin ${ }^{2}$ \\ ${ }^{1}$ Institut für Pharmazeutische Chemie der Technischen Universität Braunschweig, \\ Beethovenstraße 55, D-38106 Braunschweig, \\ ${ }^{2}$ Institut für Pharmazeutische Chemie und Pharmazeutische Technologie der Karl- \\ Franzens-Universität Graz, Schubertstraße 1, A-8010 Graz
}

\section{Zusammenfassung}

Erhitzen von Amiloridhydrochlorid $(1 \cdot \mathrm{HCl})$ in Acetatpuffer $p \mathrm{H} 4.6$ führte zu Amiloridacetat (1 - AcOH), dem Pyrazindiamin 6 und dem Acylureid 7. Das Pteridinon 3 wurde beim Erhitzen von $1 \cdot \mathbf{H C l}$ in DMF unter Rückfluß erhalten.

\section{Schlagwörter}

Amiloridhydrochlorid; Abbauprodukte; 6-Chlor-2,7-diamino-4(3H)Pteridinone.

\section{Einleitung}

Nach Mazzo [1] entstehen aus dem kaliumsparenden Diureticum Amiloridhydrochlorid (1 - $\mathrm{HCl}$ ) [2] in wässriger Lösung bei erhöhten Temperaturen und verschiedenen $\mathrm{pH}$-Werten drei Abbauprodukte. Durch saure Hydrolyse bei $\mathrm{pH}<1$ überwiegt die 6-Hydroxy-Verbindung 2 [3], bei $p H$ $\sim 5$ sind das Pteridin-Derivat 3 und die Carbonsäure 4 [4] vorhanden, 
während in alkalischer Lösung $(\mathrm{pH}>13)$ das Anion von 4 vorherrscht (Schema 1).<smiles>NC(N)=NC(=O)c1nc(Cl)c(O)nc1N</smiles>

2<smiles>C[C+](C)C(C)Cl</smiles><smiles>N</smiles><smiles>CN1N=C(N)NC(=O)c2nc(Cl)c(N)nc21</smiles>

3<smiles>CN=[N+]([NH])C(=O)Nc1nc(Cl)c(N)nc1N</smiles>

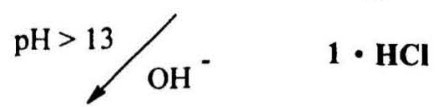<smiles>Nc1nc(N)c(C(=O)[O-])nc1Cl</smiles><smiles>C[CH+]C</smiles><smiles>Nc1nc(N)c(C(=O)O)nc1Cl</smiles>

4

Schema 1: Abbauprodukte von Amiloridhydrochlorid $(1 \cdot \mathrm{HCl})$ in wässriger Lösung [1]

Spektroskopische und analytische Daten für das Pteridinon 3 liegen nicht vor. Unser Interesse an dem Pteridinon 3 wurde ursprünglich aus einem anderen Grunde geweckt. Wir vermuteten, daß es sich bei dem blau fluoreszierenden Produkt der Farbreaktion von Amiloridhydrochlorid Ph. Eur. [5] um diese Substanz handelt. Amilorid [2] wird jedoch durch Hypobromit 
zum 3-(3-Amino-1,2,4-oxadiazol-5-yl)-5-chlor-2,6-pyrazindiamin dehydriert (Schema 2).<smiles></smiles>

Schema 2: Farbreaktion von Amilorid (1) nach Ph. Eur. [6]

Wir haben nun die Untersuchungen zur Stabilität von Amiloridhydrochlorid im schwach saurem Milieu wiederholt, um das Pteridinon 3 in die Hand zu bekommen.

\section{Ergebnisse und Diskussion}

Amiloridhydrochlorid $(1 \cdot \mathrm{HCl})$ wurde in einem Acetatpuffer $\mathrm{pH} 4.6$ unter Rückfluß erhitzt. Nach dem Abkühlen kristallisierte eine bräunliche Substanz aus, die bei der DC mit verschiedenen Eluenten das gleiche Fließverhalten wie das Edukt zeigte. Auch im El-MS stimmte der Molpeak und das Fragmentierungsschema mit dem der Base Amilorid (1) überein. Wegen der Schwerlöslichkeit der Substanz ließ sich kein ${ }^{1} \mathrm{H}-\mathrm{NMR}-\mathrm{Spektrum}$ anfertigen. Im IR-Spektrum der Substanz konnten jedoch beim Vergleich mit dem von 1 - $\mathrm{HCl}$ Unterschiede festgestellt werden. Die Elementaranalyse bewies, daß die Verbindung als Amiloridacetat (1 $-\mathrm{AcOH})$ vorlag.

Das Filtrat zeigte das DC zwei Abbauprodukte, von denen sich die lipophilere Substanz mit Chloroform aus alkalischer Lösung abtrennen ließ. Es handelt 
sich bei dieser Verbindung um das 3-Chlor-2,6-pyrazindiamin (6). Im ${ }^{1} \mathrm{H}$ NMR-Spektrum werden drei Singuletts für das $3-\mathrm{H}$ und die jeweils 2 Protonen der beiden Aminogruppen registriert. Im ${ }^{13} \mathrm{C}-\mathrm{NMR}-S p e k t r u m$ treten vier Signale für C-2, C-3, C-5 und C-6 auf. Ihre chemischen Verschiebungen entsprechen annähernd den Werten, die durch Inkrementrechnungen ausgehend von Pyrazin $(\delta=145.3)$, unter Verwendung der Inkremente für substituierte Pyridine [7], berechnet wurden. Zudem sind die Werte für die quartären Kohlenstoffe $\mathrm{C}-2$ und $\mathrm{C}-3$ mit jenen von 3-Chlor-5-iod-2,6pyrazindiamin [8] vergleichbar.

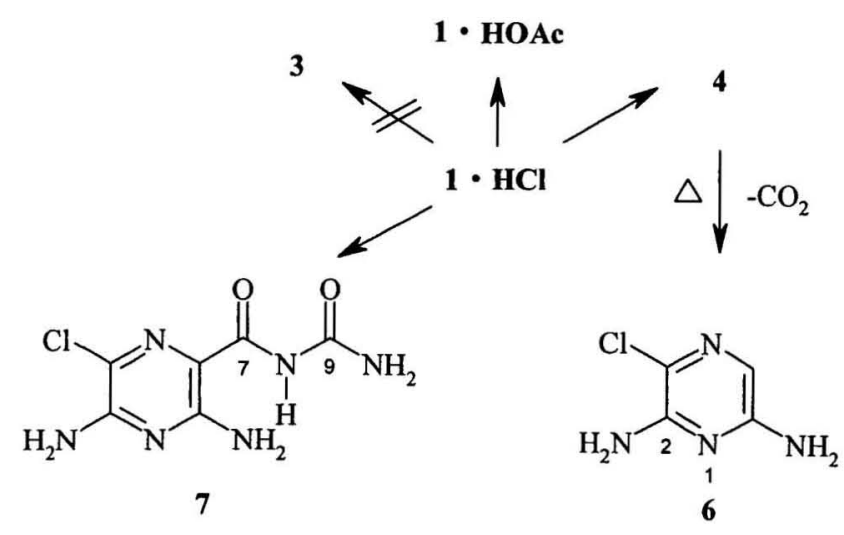

Schema 3: Abbauprodukte von Amiloridhydrochlorid $(\mathbf{1} \cdot \mathrm{HCl})$ im Acetatpuffer pH 4.6

Im El-MS ist neben dem Molpeak nach Abspaltung von Cyanwasserstoff ein weiterer intensitätsstarker Peak für $\mathrm{M}^{+}-27$ zu erkennen. Um von $1 \cdot \mathrm{HCl}$ zum Pyrazin 6 zu gelangen, muß Hydrolyse zur 3,5-Diamino-6-chlor-2pyrazincarbonsäure (4) mit anschließender Decarboxylierung erfolgt sein (Schema 3). 
Das hydrophilere Produkt wurde bei $p H \quad 6$ mit tert-Butylmethylether ausgeschüttelt und als $N$-[(3,5-Diamino-6-chlor-2-pyrazinyl)carbonyl]harnstoff (7) identifiziert. Das Hydrolyse-produkt 7 ist literaturbekannt [9]. Das ${ }^{1} \mathrm{H}$ NMR-Spektrum zeigt drei $\mathrm{NH}$-Signale im Verhältnis von $1: 1: 5$. Die ${ }^{13} \mathrm{C}$ NMR-Daten stimmen mit berechneten Werten [10] bzw. mit denen von Amilorid (1) gut überein. Im El-MS ( $\mathrm{M}^{+}$bei 230/232) wird die Seitenkette unter Eliminierung von Ammoniak zum 3,5-Diamino-6-chlor-2pyrazincarbonyl-isocyanat $(\mathrm{m} / \mathrm{z} 213 / 215)$ abgebaut. Andererseits wird unter Abspaltung von Isocyansäure das Fragment $m / 2$ 187/189 für 3,5-Diamino-6chlor-2-pyrazincarboxamid erhalten. Weitere Fragmentionen sind mit denen von Amilorid (1) identisch [6].

$$
\begin{aligned}
& \underset{\Delta}{\stackrel{\mathrm{MeOH}}{\longrightarrow}}
\end{aligned}
$$

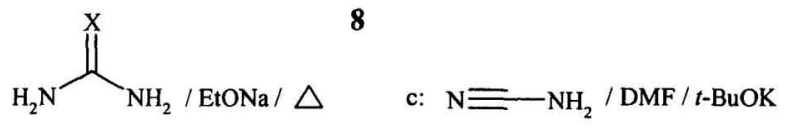

$$
\begin{aligned}
& \text { a: } X=0 \quad \text { b: } X=S \\
& \text { 1. HCl } \underset{\triangle}{\stackrel{\mathrm{DMF}}{\longrightarrow}} \text { 3 }
\end{aligned}
$$

Schema 4: Versuche zur Synthese des Pteridinons 3

Zur gezielten Darstellung des Pteridinons 3 wurden Versuche zur thermischen Cyclisierung der Amilorid-Base (1) [2] vorgenommen:

a. trockenes Erhitzen in der Mikrowelle,

b. Erhitzen in n-Butanol, auch in Gegenwart von Kalium-tert-butanolat und

c. Erhitzen in Eisessig.

Stets wurde eine nicht trennbare Produktpalette erhalten. 
Beim Erhitzen in Methanol wurde dagegen der Methylester 8 [2,11] rein isoliert. Um die Reaktivität des Esters zu prüfen, wurde sowohl mit Harnstoff /Natriumethanolat als auch mit Thioharnstoff/Natriumethanolat und Ethanol als Lösemittel erhitzt. Der gebildete gelbliche Niederschlag war wasserlöslich. Die nach Zugabe von Salzsäure ausgefällte Substanz erweis sich als 3,5-Diamino-6-chlor-2-pyrazincarbonsäure (4) [4,11]. Nach Umsetzung des Esters 8 mit Cyanamid in Dimethylformamid (DMF) und Kalium-tert-butanolat als Katalysator konnte anstelle des Pteridinons 3 ebenfalls nur die Säure 4 isoliert werden (Schema 4).

Daraufhin wurde Amiloridhydrochlorid $(1 \cdot \mathrm{HCl})$ in DMF unter Rückfluß erhitzt. Aus der heißen Lösung fiel eine gelbe Substanz aus, die im El-MS den für das Pteridinon 3 geforderten Molpeak m/z 212/214 zeigte. Unter Abspaltung eines Carbodiimid-Radikals wird mit $\mathrm{m} / \mathrm{z} 171 / 173$ ein Pyrazincabonylium-Fragment gebildet. Wegen der Schwerlöslichkeit der Verbindung 3 konnte das ${ }^{13}$ C-NMR-Spektrum nur in deuterierter Trifluoressigsäure (D-TFA) aufgenommen werden. Die Auswertung des Spektrums erfolgte durch Vergleich mit den Spektren von 2-Amino-4(3H)pteridinon (9) und 2-Amino-6,7-dimethyl-4(3H)-pteridinon (10) [12], die ebenfalls in D-TFA vermessen wurden. Bei Pterinen erfolgt in TFA Protonierung an $\mathrm{N}-1$ [13]. Das ${ }^{13} \mathrm{C}-\mathrm{NMR}$-Spektrum des Pteridinons 3 zeigt 6 quartäre Kohlenstoffatome und die gefundenen chemischen Verschiebungen für C-2, C-4 und C-8a stimmen gut mit den Verbindungen 9 und 10 überein.

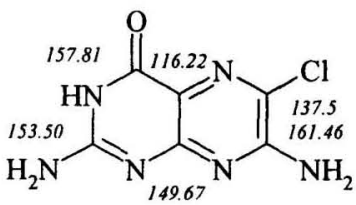

3

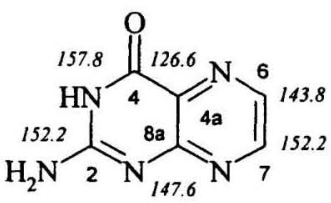

9

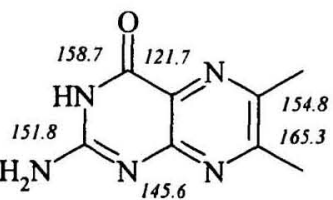

10

Schema 5: ${ }^{13} \mathrm{C}$-Verschiebungen (kursiv) von 3 und den Referenzsubstanzen 9 und 10 
Berechnet man die ungefähren chemischen Verschiebungen für C-4a, C-6, $\mathrm{C}-7$ und $\mathrm{C}-8 \mathrm{a}$ von 3 , ausgehend von den korrespondierenden Werten beim Vergleichskörper 9 unter Verwendung der Inkremente für $\mathrm{Cl}$ und $\mathrm{NH}_{2}$ bei Pyridin [7], so zeigt sich, daß auch diese Werte $(116.6 ; 130.4 ; 164.2 ; 145.5)$ gut mit den bei 3 gefundenen übereinstimmen (vgl. Schema 5).

Der endgültige Strukturbeweis für 3 durch Röntgenkristallanalyse schlug fehl, da der aus DMSO gewonnene Einkristall sich als nicht messbarer Zwilling herausstellte.

Wurde Amiloridhydrochlorid $(1 \cdot \mathrm{HCl})$ in Salzsäure $(1 \mathrm{~mol} / \mathrm{l})(\mathrm{pH} 0)$ unter Rückfluß erhitzt, so fiel nach einigen Stunden aus der siedenen Lösung eine gelbliche kristalline Substanz aus, die als Hydrochlorid des 5Hydroxypyrazin-Derivats 2 identifiziert wurde. $2 \cdot \mathrm{HCl}$ dürtte wie $1 \cdot \mathrm{HCl}$ in der Acylamino-Struktur vorliegen. Das ${ }^{1} \mathrm{H}-\mathrm{NMR}$-Spektrum in $\left[\mathrm{D}_{18}\right] \mathrm{HMPT}$ zeigt fünf Singuletts für die NH-Protonen, während das Resonanzsignal des sauren Protons der Hydroxyl-Gruppe $\left(\mathrm{pk}_{\mathrm{a}}=5.45\right.$ [13]) im Rauschpegel verschwindet. Die Signalzuordnung im ${ }^{13} \mathrm{C}-\mathrm{NMR}$-Spektrum wurde durch Vergleich mit den ${ }^{13} \mathrm{C}-N M R-D a t e n$ von Amiloridhydrochlorid (1 • HCI) [6] vorgenommen. Die Signale für C-5 und C-9 zeigen die gleiche chemische Verschiebung (Schema 6).
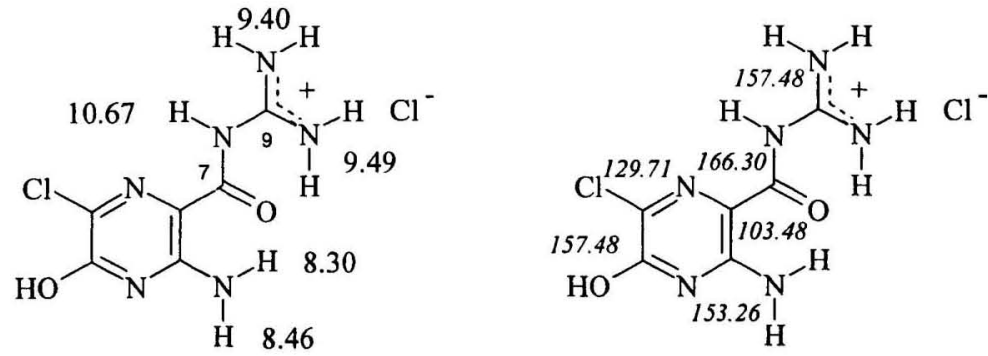

Schema 6: ${ }^{1} \mathrm{H}$ - und ${ }^{13} \mathrm{C}$ (kursiv)-Verschiebungen von $2 \cdot \mathbf{H C l}$ 
Das El-MS von $2 \cdot \mathrm{HCl}$ zeigt neben dem Molpeak von $\mathrm{m} / \mathrm{z}$ 230/232 für die Base ein dem 1 $1 \mathrm{HCl}$ entsprechendes Fragmentierungsschema [6], deren Peaks aufgrund des Austausches der 5-Amino- gegen die 5-HydroxylGruppe um jeweils eine Masseneinheit größer sind.

Mehrstündiges Erhitzen von $1 \cdot \mathbf{H C l}$ in Natronlauge $(\mathrm{pH} 13.5)$ ergab nach Ansäuern die Carbonsäure 4, wie die spektroskopischen Daten [2] zeigten.

\section{Experimenteller Teil}

Allgemeine Angaben und Geräte: [6]. * bedeutet: Zuordnung nicht gesichert.

N-[(3-Amino-6-chlor-5-hydroxy-2-pyrazinyl)carbonyl]guanidinhydrochlorid (2 . $\mathrm{HCl})$

$0,3 \mathrm{~g}(1 \mathrm{mmol}) 1 \cdot \mathrm{HCl}$-Dihydrat werden in $50 \mathrm{ml} 1 \mathrm{~mol} / \mathrm{l} \mathrm{HCl} 24 \mathrm{~h}$ unter Rückfluß erhitzt. Nach dem Abkühlen wird der Niederschlag abfiltriert, mit $\mathrm{EtOH}$ und $\mathrm{Et}_{2} \mathrm{O}$ gewaschen. Ausbeute: $230 \mathrm{mg}$ (87\%). Gelbes Pulver, Fp. > $350^{\circ} \mathrm{C},>300^{\circ} \mathrm{C}[3] .{ }^{1} \mathrm{H}-\mathrm{NMR}\left(\left[\mathrm{D}_{18}\right] \mathrm{HMPT}\right): \delta(\mathrm{ppm})=10.67$ (s, NH), 9.49 (s, $2 \mathrm{H}), 9.40(\mathrm{~s}, 2 \mathrm{H}), 8.46(\mathrm{~s}, 1 \mathrm{H}), 8,30(\mathrm{~s}, 1 \mathrm{H}) .{ }^{13} \mathrm{C}-\mathrm{NMR}\left(\left[\mathrm{D}_{18}\right] \mathrm{HMPT}\right): \delta(\mathrm{ppm})=$ 103.48 (C-2), 129.71 (C-6), 153.26 (C-3), 157.48 (C-5, C-9), 166.3 (C-7). MS $(E I): m / z(\%)=230(25)[M]^{+*}, 213(100) . I R(K B r): \tilde{v}=3438 \mathrm{~cm}^{-1}, 3398$, 3307, $3041(\mathrm{OH}, \mathrm{NH}), 1676(\mathrm{C}=\mathrm{O}), 1621, \quad(\mathrm{C}=\mathrm{N}, \mathrm{C}=\mathrm{C}) . \mathrm{CHN}$ für $\mathrm{C}_{6} \mathrm{H}_{7} \mathrm{CIN}_{6} \mathrm{O}_{2}$ - $\mathrm{HCl}$ (267.1). Ber: C 26.98 H 3.02 N 31.47; Gef: C 26.96 H 2.92 N 31.01 .

\section{2,7-Diamino-6-chlor-4(3H)-pteridinon (3)}

$0,3 \mathrm{~g}(1 \mathrm{mmol}) 1 \cdot \mathrm{HCl}-D i h y d r a t$ werden in $20 \mathrm{ml}$ DMF $8 \mathrm{~h}$ unter Rückfluß erhitzt. Der Niederschlag wird abfiltriert, mit DMF, $\mathrm{H}_{2} \mathrm{O}$ und $\mathrm{MeOH}$ gewaschen. Ausbeute: $40 \mathrm{mg}(19 \%)$. Gelbes Pulver, Fp. $>350{ }^{\circ} \mathrm{C}$. ${ }^{1} \mathrm{H}-\mathrm{NMR}$ ([D $\left.\left.\mathrm{D}_{6}\right] \mathrm{DMSO}\right): \delta(\mathrm{ppm})=6.64\left(\mathrm{~s}, 2 \mathrm{H}, \mathrm{NH}_{2}\right), 7.40\left(\mathrm{~s}, 2 \mathrm{H}, \mathrm{NH}_{2}\right), 10.89(\mathrm{~s}, 1 \mathrm{H}$, 
NH). ${ }^{13} \mathrm{C}-N M R(D-T F A): \delta(p p m)=116.22(\mathrm{C}-4 a), 137.20(\mathrm{C}-6), 149.67(\mathrm{C}-$ 8a), 153.50 (C-2), 157.81 (C-4), 161.46 (C-7). MS (EI): m/z (\%)= 212 (100) $[\mathrm{M}]^{+\bullet}$. IR $(\mathrm{KBr}): \tilde{v}=3412 \mathrm{~cm}^{-1}(\mathrm{NH}), 3359,3150\left(\mathrm{NH}_{2}\right), 1690(\mathrm{C}=0), 1618$ $(C=N, C=C)$. HPLC (MeCN/H $\left.H_{2} \mathrm{O} 20: 80\right): t_{s}=0.03$ min. $\mathrm{CHN}$ für $\mathrm{C}_{6} \mathrm{H}_{5} \mathrm{CIN}_{6} \mathrm{O}$ (212.6). Ber: C 33.90 H 2.37 N 39.53; Gef: C 33.03 H 2.30 N 35.91.

\section{N"[(3,5-diamino-6-chlor-2-pyrazinyl)carbonyl]guanidin acetat (1 - HOAc)}

$0,91 \mathrm{~g}(3 \mathrm{mmol}) 1 \cdot \mathrm{HCl}$-Dihydrat werden in $120 \mathrm{ml} \mathrm{H} \mathrm{H}_{2} \mathrm{O}$ und $30 \mathrm{ml}$ AcOH/AcONa-Puffer $p \mathrm{H}$ 4,6 unter Rückfluß 24 h erhitzt. Nach dem Abkühlen wird der Niederschlag abgesaugt. Ausbeute: $160 \mathrm{mg}$ (18\%). Braune Kristalle, Fp. ab $240{ }^{\circ} \mathrm{C}$ (Zers.). MS (El): $\mathrm{m} / \mathrm{z}(\%)=229(100)[\mathrm{M}]^{+\bullet}, 212(20)$. IR (KBr): $\tilde{v}=3400 \mathrm{~cm}^{-1}, 3337,3060(\mathrm{NH}), 1708(\mathrm{C}=0), 1667,1620(\mathrm{C}=\mathrm{N}, \mathrm{C}=\mathrm{C}) . \mathrm{CHN}$ für $\mathrm{C}_{6} \mathrm{H}_{8} \mathrm{ClN} 7 \mathrm{O} \cdot \mathrm{CH}_{3} \mathrm{COOH}$ (289.6). Ber: C $33.17 \mathrm{H} 4.18 \mathrm{~N} 33.85$; Gef: C $33.08 \mathrm{H} 4.17$ N 33.61 .

\section{3-Chlor-2,6-pyrazindiamin (6)}

Das Filtrat von $1 \cdot$ HOAc wird mit Natronlauge alkalisiert und $3 \times$ mit 70 $\mathrm{ml} \mathrm{CHCl}{ }_{3}$ ausgeschüttelt. Die organische Phase wird über $\mathrm{Na}_{2} \mathrm{SO}_{4}$ getrocknet und i. Vak. abgezogen. Ausbeute: $30 \mathrm{mg}$ (7\%). Gelbe Nadeln, Fp. $170{ }^{\circ} \mathrm{C}$ (Toluol). ${ }^{1} \mathrm{H}-\mathrm{NMR}$ ([D $\left.\left.\mathrm{D}_{6}\right] \mathrm{DMSO}\right): \delta(\mathrm{ppm})=6.86(\mathrm{~s}, 5-\mathrm{H}), 6.07\left(\mathrm{~s}, \mathrm{NH}_{2}\right), 5.98(\mathrm{~s}$, $\left.\mathrm{NH}_{2}\right) .{ }^{13} \mathrm{C}-N M R\left(\left[\mathrm{D}_{6}\right] \mathrm{DMSO}\right): \delta(\mathrm{ppm})=116.74$ (C-5), 117.29 (C-3), 150.21 (C-6), 154.23 (C-2). MS (El): m/z (\%)= $144(100)[M]^{+\bullet}, 117(50)$. IR (KBr): $\tilde{v}$ $=3467 \mathrm{~cm}^{-1}, 3308,3154(\mathrm{NH}), 1644,1614(\mathrm{C}=\mathrm{N}, \mathrm{C}=\mathrm{C}) . \mathrm{CHN}$ für $\mathrm{C}_{4} \mathrm{H}_{5} \mathrm{CIN}_{4}$ (144.5). Ber: C 33.23 H 3.48 N 38.76; Gef: C 33.77 H 3.38 N 37.57.

\section{N-[(3,5-Diamino-6-chlor-2-pyrazinyl)carbonyl]harnstoff (7)}

Die verbleibende wässrige Phase von Substanz 6 wird auf $\mathrm{pH} 6$ eingestellt und $3 \times$ mit tert-BuOMe ausgeschüttelt. Nach Trocknen über 
$\mathrm{Na}_{2} \mathrm{SO}_{4}$ wird das Lösungsmittel i. Vak. abdestilliert. Ausbeute: $14 \mathrm{mg}(2 \%)$, Fp. $260^{\circ} \mathrm{C}(\mathrm{MeOH})$ [9]. ${ }^{1} \mathrm{H}-\mathrm{NMR}\left(\left[\mathrm{D}_{6}\right] \mathrm{DMSO}\right): \delta(\mathrm{ppm})=8.84(\mathrm{~s}, 1 \mathrm{H}), 7.68(\mathrm{~s}$, $1 \mathrm{H}), 7.33(\mathrm{~s}, 5 \mathrm{H}) .{ }^{13} \mathrm{C}-\mathrm{NMR}\left(\left[\mathrm{D}_{6}\right] \mathrm{DMSO}\right): \delta(\mathrm{ppm})=109.67(\mathrm{C}-2), 118.68(\mathrm{C}-$ 6), 152.95 * (C-3), 153.93 * (C-5), 155.37 * (C-9), 164.92 (C-7). MS (EI): m/z $(\%)=230(30)[\mathrm{M}]^{+\bullet}, 213(100) . I R(\mathrm{KBr}): \tilde{v}=3466 \mathrm{~cm}^{-1}, 3328,3153(\mathrm{NH})$, $1727,1671(\mathrm{C}=\mathrm{O}), 1637,1617(\mathrm{C}=\mathrm{N}, \mathrm{C}=\mathrm{C})$.

\section{Methyl-3,5-diamino-6-chlor-2-pyrazincarboxylat (8)}

$0,23 \mathrm{~g} \mathrm{(1} \mathrm{mmol}) 1$ werden in einer Mischung aus $25 \mathrm{ml} \mathrm{CHCl}_{3}$ und $25 \mathrm{ml}$ $\mathrm{MeOH} 17 \mathrm{~h}$ unter Rückfluß erhitzt. Es wird mit $50 \mathrm{ml}$ Wasser und anschließend $4 \times$ mit $50 \mathrm{ml} \mathrm{CHCl}_{3}$ ausgeschüttelt. Die vereinigten organischen Phasen werden über $\mathrm{Na}_{2} \mathrm{SO}_{4}$ getrocknet und i. Vak. abdestilliert. Ausbeute: $120 \mathrm{mg}(60 \%)$. Gelbe Nadeln, Fp. $207^{\circ} \mathrm{C}(\mathrm{MeOH}), 212-213^{\circ} \mathrm{C}$ $(\mathrm{MeCN})$ [2]. ${ }^{1} \mathrm{H}-\mathrm{NMR}\left(\left[\mathrm{D}_{6}\right] \mathrm{DMSO}\right): \delta(\mathrm{ppm})=3.73\left(\mathrm{~s}, \mathrm{CH}_{3}\right), 7.10(\mathrm{~s}, 2 \mathrm{H}), 7.20$ $(\mathrm{s}, 2 \mathrm{H}) .{ }^{13} \mathrm{C}-N M R\left(\left[\mathrm{D}_{6}\right] \mathrm{DMSO}\right): \delta(\mathrm{ppm})=51.1(\mathrm{C}-8), 109.4(\mathrm{C}-2), 119.2(\mathrm{C}-6)$, 153.2 * (C-5), 155.8 * (C-3), 165.8 (C-7). MS (EI): m/z (\%)= 202 (100) [M] ${ }^{+\bullet}$, 144 (75). IR (KBr): $\tilde{v}=3477 \mathrm{~cm}^{-1}, 3449,3355,3150(\mathrm{~N}-\mathrm{H}), 1669(\mathrm{C}=\mathrm{O})$,. 1644, 1605 ( $C=N, C=C)$. UV (Dioxan): $\lambda$ max $(\lg \varepsilon)=269 \mathrm{~nm}$ (4.19), 351 (4.16). Fluoreszenz (Dioxan/Anregungswellenlänge $270 \mathrm{~nm}$ ): $\lambda_{\max }=400 \mathrm{~nm}$. $\mathrm{CHN}$ für $\mathrm{C}_{6} \mathrm{H}_{7} \mathrm{ClN}_{4} \mathrm{O}_{2}$ (202.6). Ber: C 35.57 H $3.48 \mathrm{~N} 27.65$; Gef: C $35.60 \mathrm{H}$ 3.45 N 27.47.

\section{3,5- Diamino-6-chlor-2-pyrazincarbonsäure (4)}

$0,23 \mathrm{~g}(1 \mathrm{mmol}) 1$ wird in $50 \mathrm{ml} \mathrm{H}_{2} \mathrm{O}$ suspendiert. Es wird mit $4 \mathrm{~mol} / \mathrm{l}$ $\mathrm{NaOH}$ auf $p \mathrm{H} 13,5$ eingestellt und $7 \mathrm{~h}$ bei $90^{\circ} \mathrm{C}$ erwärmt. Nach dem Abkühlen wird angesäuert, der Niederschlag abgesaugt und mit $\mathrm{H}_{2} \mathrm{O}$, wenig EtOH abs. und $\mathrm{Et}_{2} \mathrm{O}$ gewaschen. Ausbeute: $110 \mathrm{mg}$ (57\%). Hellgelbes Pulver, Fp. 225 
${ }^{\circ} \mathrm{C}\left(\mathrm{DMSO} / \mathrm{H}_{2} \mathrm{O}\right), 230-231{ }^{\circ} \mathrm{C}, 272{ }^{\circ} \mathrm{C}\left(\mathrm{DMSO} / \mathrm{H}_{2} \mathrm{O}\right)$ [4]. ${ }^{1} \mathrm{H}-\mathrm{NMR}\left(\left[\mathrm{D}_{6}\right] \mathrm{DMSO}\right)$ : $\delta(\mathrm{ppm})=12.13(\mathrm{~s}, \mathrm{OH}), 7.21(\mathrm{~s}, 4 \mathrm{H}) \cdot{ }^{13} \mathrm{C}-\mathrm{NMR}\left(\left[\mathrm{D}_{6}\right] \mathrm{DMSO}\right): \delta(\mathrm{ppm})=$ 110.1(C-2), 118.7 (C-6), 153.2 * (C-5), 155.9 * (C-3), 167.3 (C-7). MS (EI): $\mathrm{m} / \mathrm{z}(\%)=188(100)[\mathrm{M}]^{+*}, 144(80) . I R(\mathrm{KBr}): \tilde{v}=3619 \mathrm{~cm}^{-1}, 3415,3317$, $3167\left(\mathrm{OH}, \mathrm{NH}_{2}\right), 1664(\mathrm{C}=\mathrm{O}), 1618,1559(\mathrm{C}=\mathrm{N}, \mathrm{C}=\mathrm{C})$. UV (Dioxan): $\lambda_{\max }$ (lg $\varepsilon)=269 \mathrm{~nm}$ (4.11), 352 (4.08). $\mathrm{CHN}$ für $\mathrm{C}_{5} \mathrm{H}_{5} \mathrm{ClN}_{4} \mathrm{O}_{2}$ (188.6). Ber: $\mathrm{C} 31.85 \mathrm{H}$ 2.67 N 29.71; Gef: C 31.93 H 2.80 N 29.46 .

\section{Literatur}

[1] Mazzo D J in Florey K, editor. Analytical Profiles of Drug Substances, Volume 15, Academic. Press, New York, 1986: 1-34.

[2] Smith R L, Cochran D W, Gund P, Cragoe, Jr. E J. Proton, Carbon-13, and Nitrogen-15 Nuclear Magnetic Resonance and CNDO/2 Studies on the Tautomerism and Conformation of Amiloride, a Novel Acylguanidine.

J. Am. Chem. Soc. 1979; 101: 191-201.

[3] Cragoe, Jr. E J, Woltersdorf, Jr. O W, Bicking J B, Kwong S F, Jones J $\mathrm{H}$.

Pyrazine Diuretics. II., N-Amidino-3-amino-5-substituted 6-Halopyrazinecarboxamides.

J. Med. Chem. 1967; 10: 66-75.

[4] Shepard K L, Mason J W, Woltersdorf, Jr. O W, Jones J H, Cragoe, Jr. E J.

Pyrazine Diuretics. VI. (Pyrazinecarboxamido)Guanidines.

J. Med. Chem. 1969; 12: 280-285.

[5] Europäisches Arzneibuch 2002. 4th ed. 
Deutscher Apotheker Verlag Stuttgart, Govi-Verlag - Pharmazeutischer Verlag GmbH Eschborn, 2002: 1169.

[6] Görlitzer K, Huth S, Jones P G, Gößnitzer E, Wendelin W. Zur Farbreaktion von Amiloridhydrochlorid Ph. Eur.

Sci. Pharm. 2001; 69: 275-287.

[7] Kalinowski H-O, Berger S, Braun S.

${ }^{13} \mathrm{C}$ NMR Spektroskopie. Georg Thieme: Stuttgart, 1984.

[8] Chapdelaine M J, Warwick P J, Shaw A.

An Efficient Method for Preparation of 3,5-Diamino-6-chlorpyrazin-2-yl Alkylketones Using a Novel Acetylene Hydrattion Method.

J. Org. Chem. 1989; 54: 1218-1221.

[9] Hanifin J W, Capuzzi R, Cohen E.

The Preparation of Substituted N-Carbamoylpyrazinecarboxamides.

J. Med. Chem. 1969; 12: 1102-1103.

[10] ACD/CNMR Predictor, Release 7.00, Product Version 7.07

[11] Tobias S, Günther H, Pfleiderer W.

Pteridin-Spektren IV.

Chem. Ber. 1985; 118: 354-362.

[12] Ewers U, Günther $H$, Jaenicke L.

Pteridin-Spektren III.

Chem. Ber. 1974; 107: 3275-3286.

[13] Bock M G, Schlegel H B, Smith G M.

Theoretical Estimation of pKa Values of Pyrazinylguanidine Derivatives. J. Org. Chem. 1981; 46: 1925-1927. 